\title{
A PROCEDURE FOR AUTOMATIC OBJECT-BASED CLASSIFICATION
}

\author{
Prashanth Reddy $\operatorname{Marpu}^{a}, \operatorname{Irmgard~Niemeyer}^{b}$, Richard Gloaguen $^{a}$ \\ ${ }^{a}$ Institute for Geology, Freiberg University of Mining and Technology, 09599, Freiberg, Germany \\ prashanthreddym@mail.com, gloaguen@geo.tu-freiberg.de \\ ${ }^{b}$ Institute for Mining and Geodesy, Freiberg University of Mining and Technology, 09599, Freiberg, Germany \\ irmgard.niemeyer@tu-freiberg.de
}

KEY WORDS: Object-based classification, automatic classification, geological faults, fission tracks

\begin{abstract}
:
\section{INTRODUCTION}

The object-based classification starts with segmentation of the image into highly homogeneous image regions (or objects). These image segments correspond to the approximations of real world objects which can be characterised by shape and texture (Benz et al, 2004). Often in image processing, the objects to be identified in the image can be visually separated based on their shape and texture details. Object-based classification techniques can thus be efficient in the context of identifying visible objects. An automatic classification procedure is prepared in this effect to minimise human involvement in classification steps. Such a procedure, speeds up the process of classification when huge data is to be dealt with, however at the expense of accuracy. We used this procedure to make algorithms for extracting morphology of geological faults using remote sensing data and in identifying fission tracks in microscopic images
\end{abstract}

Object-based classification is a promising methodology. Unlike pixel-based techniques which only use the layer pixel values, the object-based techniques can also use shape and context information of a scene texture. These extra degrees of freedom provided by the objects will aid the identification of visible textures. In this article, we present a procedure for object-based automatic classification. Using this procedure, we made algorithms to classify the geological faults using remote sensing data and fission-tracks in microscopic images. We also show how the notion of the object makes the task easy to use the result of classification for varius studies.

\section{METHODOLOGY}

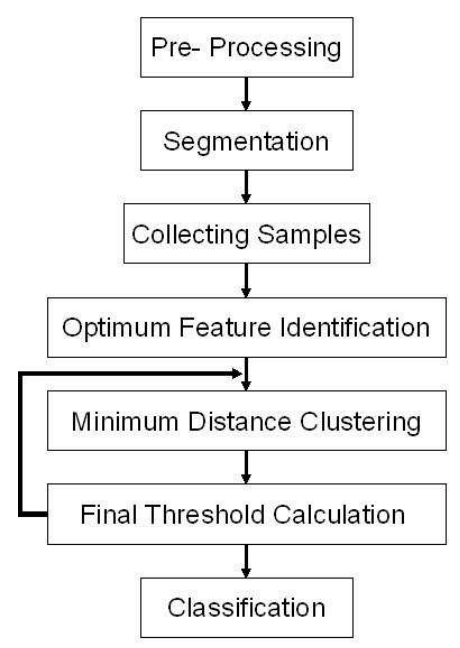

Figure 1: The Procedure for Object-based Classification

The flowchart shown in Fig. 1 illustrates the procedure. The aim is to classify the image into two classes i.e. the object class of interest and the background. Based on the above procedure we can make algorithms for different cases, by using different techniques in the different stages of the procedure. In this article we present algorithms that are based on the assumption that all the random variables encountered during the processing can be approximated as Gaussian distributions.

1. Preprocessing is an optional step. It is to ensure proper segmentation (dividing the image in to small groups of pixels) by means of creating extra image layers or enhancing existing image layers. In some cases using averaging filter on the image can help to extract better image objects. Different image scenario will require different pre-processing steps. Processing a radar image requires that the speckle has been minimised, whereas processing a Digital Elevation Model (DEM) to extract lineaments and faults necessitates that a derivative image has been prepared. Sometimes the pre-processing step can be crucial as the basic step of object-based classification is to first generate approximate real world objects and it requires that these object regions in the image are homogeneous.

2. After segmenting the image into primitive objects, a few samples are collected. This can be done manually by selecting samples in the image based on human interpretation or statistically by selecting specific regions in the concerned image histograms. For example if the objects of class of interest are characterised by bright regions compared to other objects in the image, then $2-5 \%$ of the objects in the image histogram which have high mean values are taken as samples of class of interest and 2-5\% of image objects which have low mean values are assigned as samples to the background class.

3. The optimal features can be identified visually by looking at the feature values graphically. Unless we have a good understanding of the type of classes and can intuit what features can be possible candidates, it can be a laborious task to identify them as the objects can be characterised by a huge number of features. When we have samples of the classes, we can automatically identify the optimal features (features are the characteristics defined for an object. e.g., mean value, standard deviation, length, width, area, etc (Baatz et al, 2004)) which can separate the two classes effectively based on Jeffries-Matusita distance, $J$ (Nussbaum 
et al, 2005). For two classes $C_{1}$ and $C_{2}$ of size $n_{1}, n_{2}$ with means $m_{1}, m_{2}$ and standard deviations $\sigma_{1}, \sigma_{2}$ respectively,

$$
B=\frac{1}{8}\left(m_{1}-m_{2}\right)^{2} \frac{2}{\sigma_{1}^{2}+\sigma_{2}^{2}}+\frac{1}{2} \ln \left(\frac{\sigma_{1}^{2}+\sigma_{2}^{2}}{2 \sigma_{1} \sigma_{2}}\right)
$$

$B$ is the Bhattacharya distance. Then,

$$
J=2\left(1-e^{-B}\right)
$$

A distance measure different to that of the Jeffries-Matusita distance can also be used in this step to identify the optimum feature space.

4. With respect to the means of the samples of two classes and in the feature space defined by the optimal features identified in the previous step, clusters are formed using the minimum distance criterion. This clustering is an approximation of the desired classification. To represent all the features on a common scale, a transformation has to be made on the feature values before clustering. This transforms all the feature values in the range of $[0,1]$. For every object feature value $F$ of a particular feature,

$$
\begin{gathered}
F_{1}=F-F_{\text {min }} \\
F^{\prime}=\frac{F_{1}}{F_{1 \max }}
\end{gathered}
$$

$F_{\min }$ is the minimum of the object feature values of that feature, $F_{1 \max }$ is the maximum of values $F_{1}$ obtained in first step. $F^{\prime}$ is the transformed feature value of $F$. A different clustering algorithm can also be used at this stage.

5. The final thresholds for features to separate the two classes is then found based on Bayes' conditional probability principle. For classes $C 1$ and $C 2$, the threshold can be found as (Nussbaum et al, 2005)

$T=\frac{m_{2} \sigma_{1}^{2}-m_{1} \sigma_{2}^{2} \pm \sigma_{1} \sqrt{\left(m_{1}-m_{2}^{2}+2 A\left(\sigma_{1}^{2}-\sigma_{2}^{2}\right)\right.}}{\left(\sigma_{1}^{2}-\sigma_{2}^{2}\right)}$

where,

$$
A=\log \left[\frac{\sigma_{1}}{\sigma_{2}} * \frac{n_{2}}{n_{1}}\right]
$$

Using this, the thresholds of features are calculated for the two classes (i.e., class of interest and the background), based on the distribution of objects classified in the previous step. The real thresholds are near these calculated thresholds. An example probability distribution of the classes can be observed in Fig. 2. From the figure, we can interpret that the thresholds should be moved further towards mean of background class so as not to mis-classify any of the objects of the class of interest. However, shifting the threshold much further can be troublesome as more objects of background class will be included for the sake of very less objects of the class of interest. Having the thresholds close to the actual thresholds found immediately after clustering rather than the mean of background class, is a safe prospect though there will be some misclassification. This can be achieved by defining a simple criterion using the separability measure. If $J<0.5$, the feature is ignored. Otherwise,

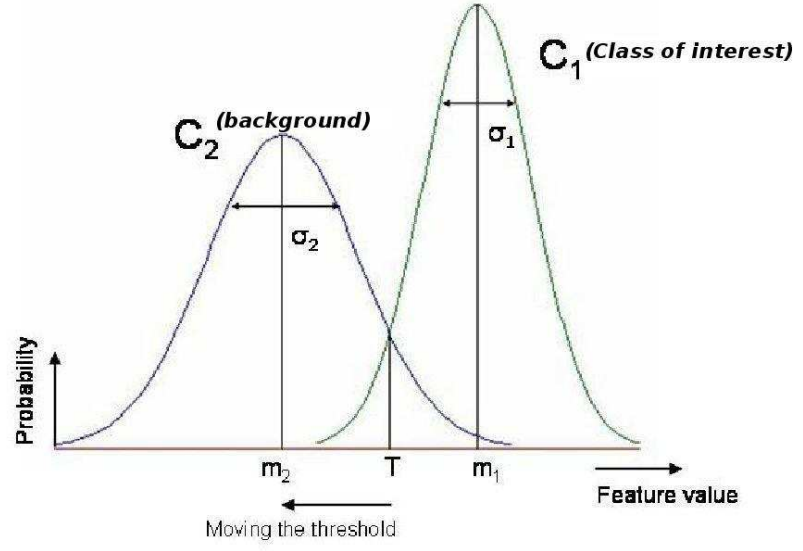

Figure 2: Finding the threshold

The above criterion is based on observations using random data. It has been observed that this criterion is suitable when more features with a better $J$ value exist. The objects are finally classified using these thresholds. The classification is based on an 'AND' operation and as enough number of features are considered with well defined thresholds, majority of the background class objects from the cluster of objects of the class of interest are reclassified as background objects and vice-versa.

\section{CASE STUDIES}

\subsection{Extracting the morphology of normal faults}

Background: Normal faults are formed in the rifts. A rift is a region where the Earth's crust and lithosphere are under extensional strain, hence forming a series of horst and graben segments. The fractures generated in this process of rifting are normal faults. Fig. 3 shows the geometry in a rift. The faults grow in time and form a network of faults which is fractal.

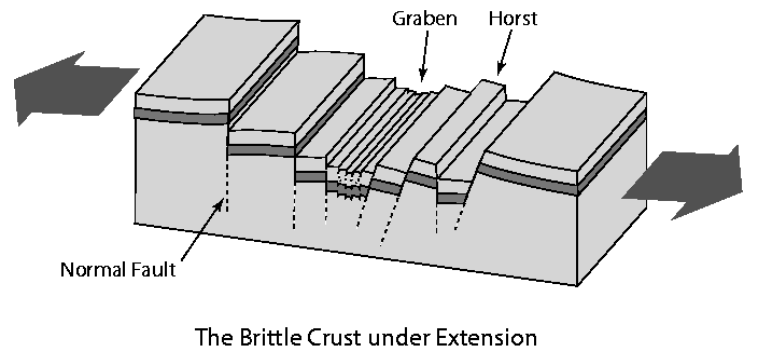

Figure 3: The fault geometry in a rift

By virtue of the geometry of a side-looking imaging radar, the same scene imaged in two opposite directions can effectively map the faults (Henderson and Lewis, 1998). A DEM can also be used for identification of the faults as the faults are characterised by steep slopes. The radar images are one of the sources, for generating a DEM and hence are the basic input in the process of fault mapping.

In this context an algorithm is prepared using the defined procedure to process the radar images and DEM separately and automatically classify the faults in the region described by the image. The algorithm is applied on the data of Lake Magadi area in South Kenya. This area is chosen as it is an active fault zone. The two 
images of the stereo pair should be used to classify the two directions of the faults and the result has to be combined to generate the final fault map. Such a fault map is essential to analyse the deformation of the region. For example, studying the fault statistics to calculate the fractal dimension gives an indirect measure to quantify the deformation of the region. The concept of the object thus helps in determining the statistics immediately after classifying as the resulting map contains objects of faults.

Algorithm and Results: The algorithms for classifying the DEM and radar image will almost be the same, except for the preprocessing step and identifying proper input parameters for segmentation. In the pre-processing step, radar images are speckle filtered and averaged to produce homogeneous textures and different approximations of derivative of a DEM are used as image layers in the case of DEM. Faults are characterised by high amplitude in radar images and high derivative values in the DEM. For the same resolution in the images, the segmentation parameters will be the same. Samples are then selected from the concerned histograms of the means of the objects. Fig. 4 shows the derivative of the DEM from Lake magadi region in south Kenya. The synthetic aperture radar (SAR) image used here is acquired using RADARSAT. Fig. 5 and Fig. 6 show the results of automatically

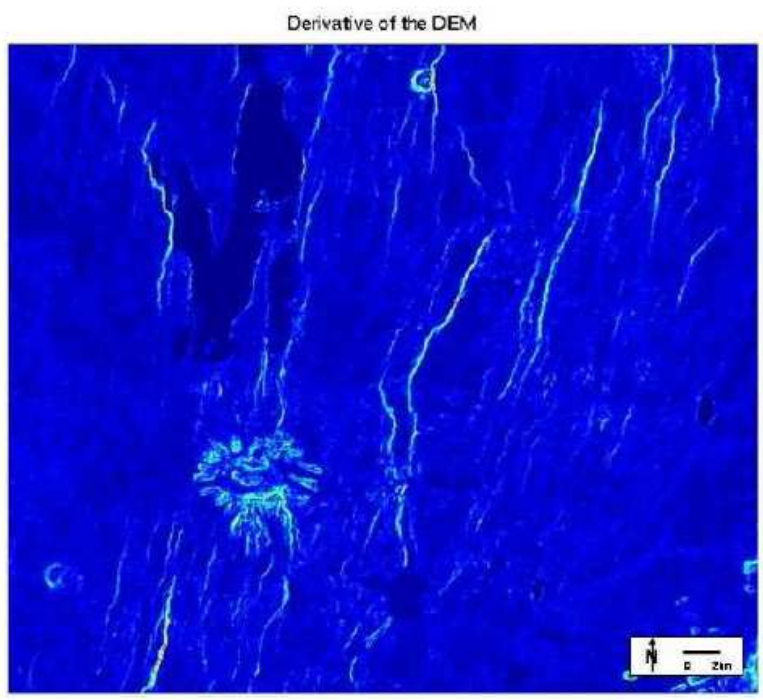

Figure 4: Derivative of the DEM in the region near Lake Magadi, South Kenya

classifying the DEM and radar image respectively. As, can be observed the result of the DEM is more convincing than the radar image. So it is suggested that the DEM should be first created out of the stereo pair and the faults are extracted from the DEM for further extending the classification to study the statistics of the faults. Fig. 5 and Fig.6 show the results of automatically classifying the DEM and radar image respectively. As, can be observed the result of the DEM is more convincing than the radar image. So it is suggested that the DEM should be first created out of the stereo pair and the faults are extracted from the DEM for further extending the classification to study the statistics of the faults.

As mentioned earlier, the notion of the object helps in the image analysis after the classification. After the classification every real world fault is a fault object in the image. So, it is easy to estimate the statistics of the faults using the faults objects in the image. The shape of the object can be used to find the approximate width and length of the faults. This statistics help in determining the fractal dimension of the faults in that region, which has many applications in geosciences (Turcotte, 1997). Fig. 7 shows the

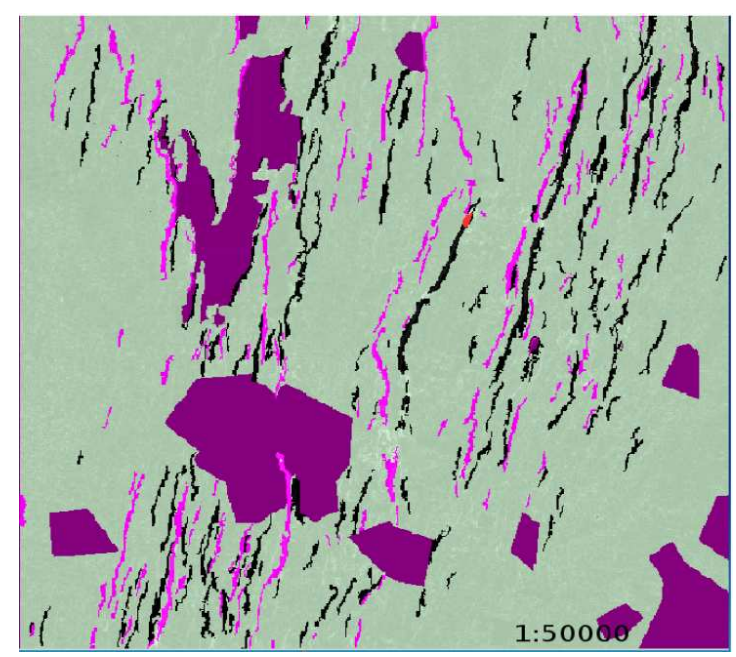

Figure 5: Faults in the DEM (unwanted areas like volcanoes are masked)

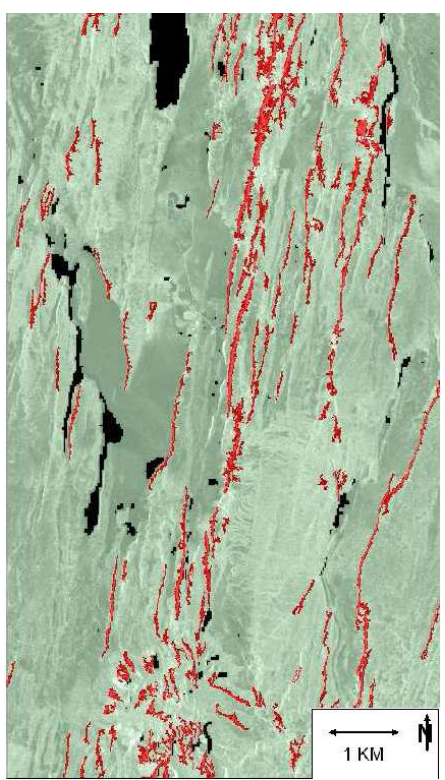

Figure 6: Classified faults (indicated as red regions) in the radar image of the Lake Magadi region, South Kenya

length distribution and the corresponding fractal dimension calculated using that information. The linearity of the curve in the $\log -\log$ plot of the cumulative histogram of lengths of faults also validates the method used in the present work. Such applications of objects after classification makes the object-based methodology a powerful tool in image analysis. 

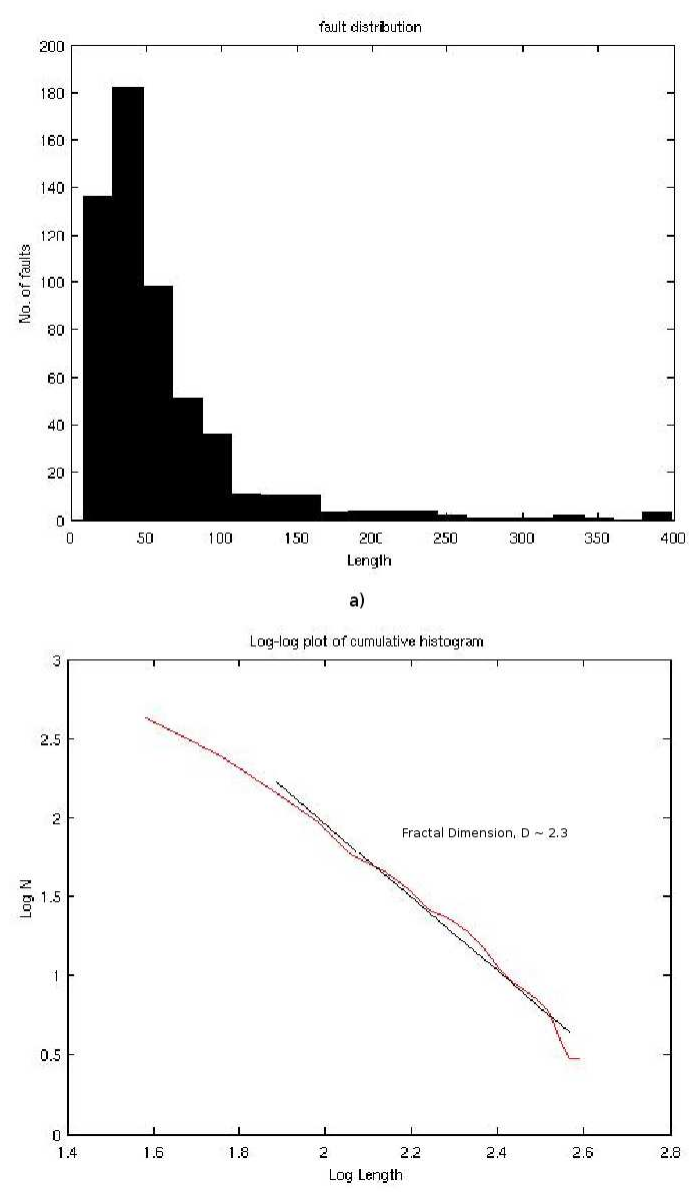

Figure 7: The histogram of the faults lengths and the corresponding cumulative histogram curve in logarthmic scale to find fractal dimension.

\subsection{Identifying Fission-tracks in Microscopic images}

Background: The fission-track dating method is now commonly used in geological research but hindered by time consuming track counts and length measurements. Attempts at automation using conventional image analysis techniques on digital data have hitherto proved of limited practical use (e.g. Morgan et al, 2003). To automate the process of counting the tracks, we try to mimic human thinking procedures. We first identify all the tracks in the image and then count the number of tracks by accounting for the number of intersections. The first step in doing that is to automatically classify the tracks and define 'tracks' objects. When the tracks are identified, then we can work on identifying procedures to count the individual tracks by counting the intersections. We used the procedure described in this article to develop an algorithm for first identifying the tracks objects in the image.

Algorithm and Results: The pre-processing step involves some image enhancement and morphological closing to generate homogeneous regions of tracks. It has been observed in several of the acquired images that the standard deviation of the pixel values of the fission-tracks pixels is very high compared to that of the background. It is therefore the objective of the pre-processing step to generate homogeneous areas by reducing the standard deviation of the fission-tracks pixels. The following transformation is applied to the image

$$
I_{1}=\sqrt{I_{\max }^{2}-I^{2}}
$$

$I_{\max }$, is the maximum value in the image $I$. The image $I_{1}$ is then inverted so as to represent the tracks pixels in dark as the above transformation inverts the image histogram by compressing the lower part of the histogram. Then, on the resulting image, morphological closing operation is performed so as to generate homogeneous regions. Fig. 9 shows the result of processing the image shown in Fig. 8 using the described method.

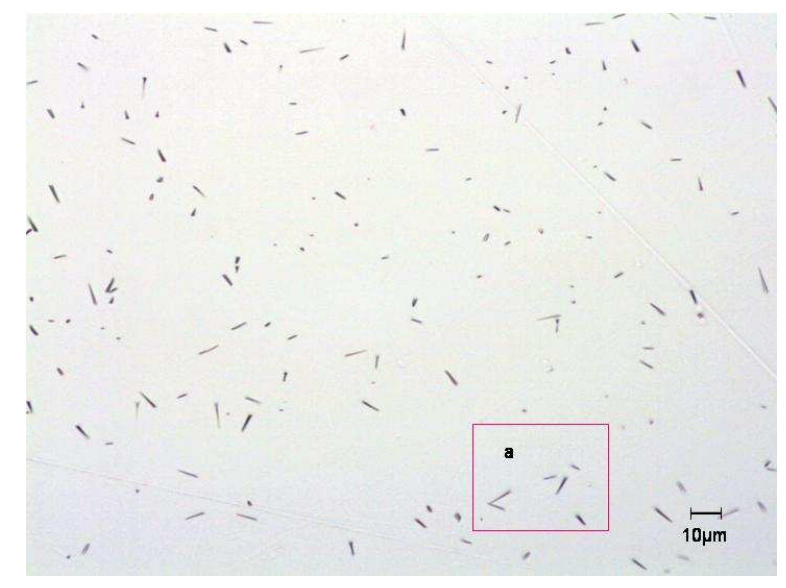

Figure 8: A microscopic image showing tracks to be identified

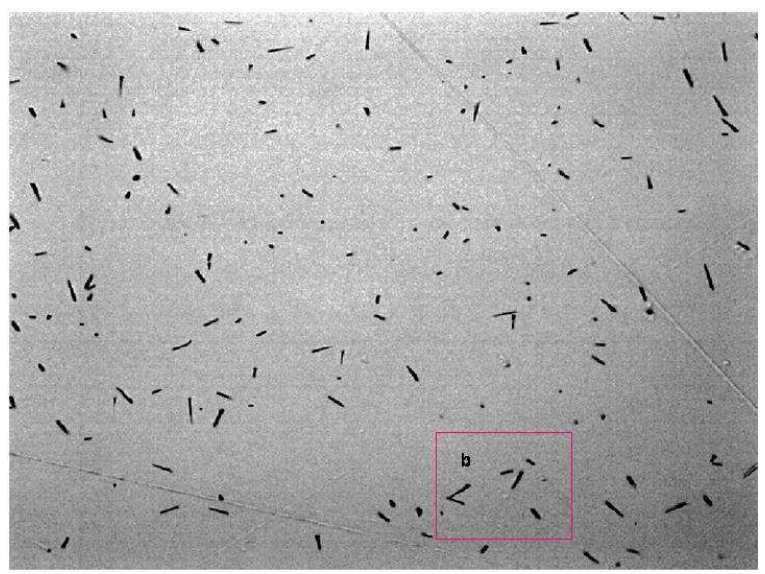

Figure 9: Processesed image using image enhancement and morphological closing

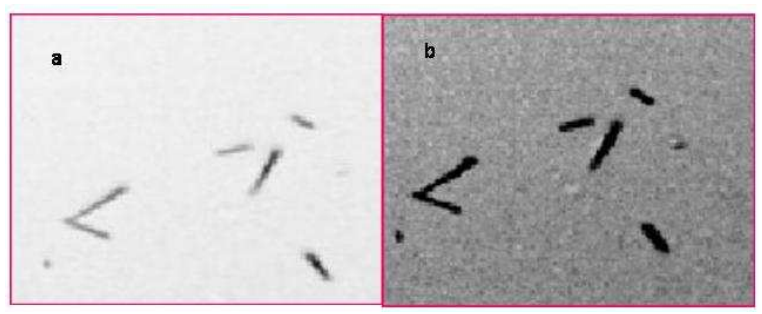

Figure 10: A comparison using a close-up. It can be observed that the tracks are more homogeneous

The rest of the algorithm is the same as in the case of faults. The same distance measure is used to find the optimal features. The segmentation parameters are to be identified separately for different images based on lens magnifications. For a set of images acquired with the same camera on the same microscope using the same lens magnification, the same set of segmentation parameters will apply. So, it is necessary to fist define standards for image acquisition so as to use the same segmentation parameters. 
Tracks are characterised with low pixel values in the image. So, objects at the lower end of the histogram of the object means are selected as samples for object class of interest i.e., tracks. And, the objects at the higher end of the histogram are taken as samples of background objects class. In the present case a microscopic image acquired with a lens magnification of 20 is used. The result of classification is shown in Fig. 11. The object-based

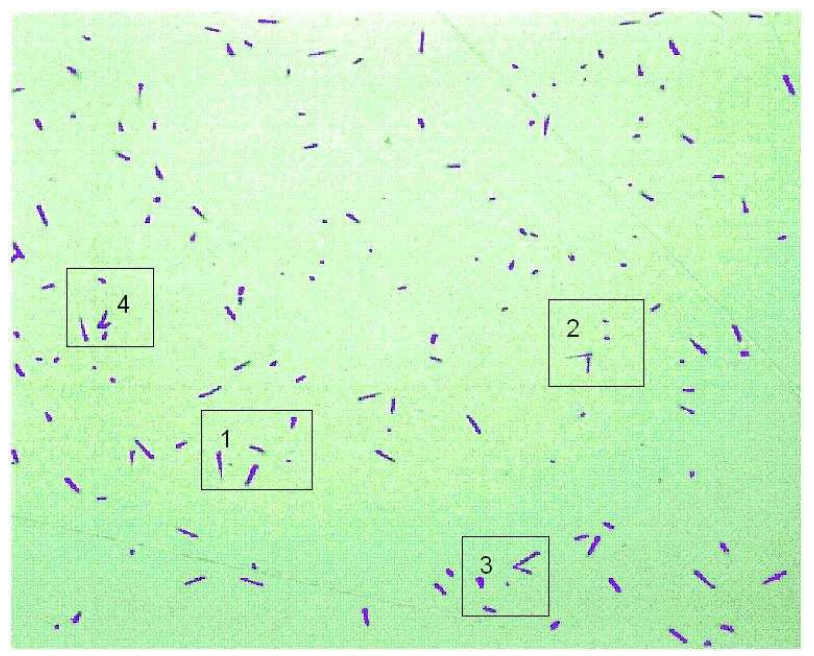

Figure 11: Classified Tracks

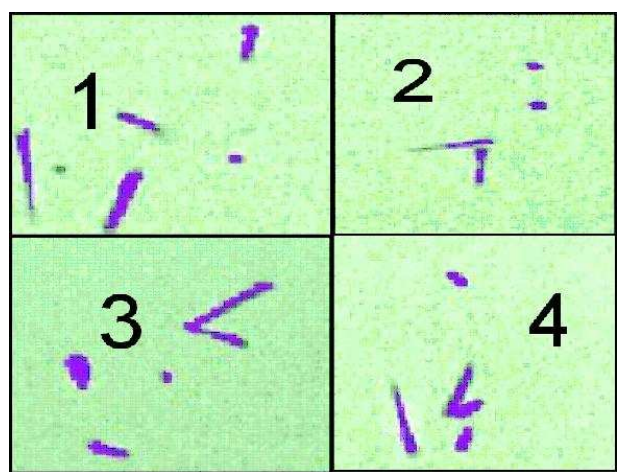

Figure 12: Zooming the regions 1,2,3,4 in the classified image. The classification is seen in magenta and green over the grey scale image.

classification extracts the shape of the tracks with a good overall accuracy. The classified objects are then ready for a different stage where the shape of the object is analysed to detect the intersections so as to count the exact number of tracks in the image. The concept of using objects for classification thus helps in using the object information directly in the next stages where the tracks are counted.

\section{CONCLUSIONS}

A procedure has been defined for object-based automatic classification. We show that the object-based classification is a powerfull tool to extract visible textures automatically. We have demonstrated on two geological examples that the proposed procedure allows an accurate counting of the objects. This results have a huge impact for the determination of stress and strain in extensional structures and the FT-dating of rocks. This method has a more general outcome for image analysis. The algorithms developed by using the procedure provide very good results with an overall accuracy of $>90 \%$. The concept of object helps in the post-classification image analysis. A gaussian approximation of all the distributions encountered has been used in this work. But it is possible as a future work to use a maximum likelihood estimator to incorporate several other distributions if appropriate. After the minimum distance clustering, we get an approximate distribution of the original classes. If the model fits the original distribution, the calculated thresholds will be more accurate.

\section{REFERENCES}

Benz, U.C., Hoffmann, P., Willhauck, G., Lingenfelder, I. and Heynen,M., Multi-resolution, object-oriented fuzzy analysis of remote sensing data for GIS-ready information, ISPRS Journal of Photogrammetry \& Remote sensing, Vol. 58, 239-258, 2004

Baatz et al, eCognition Userguide,2004. URL: http://www.definiens-imaging.com.

Henderson, F.M., and Lewis, A.J., Principles \& Applications of imaging Radar, manual of Remote sensing,3rd edition, Vol.2. John Wiley \& sons, 1998.

Morgan, L., Meehan, Q. and Stewart, R., Fission-track dating using digital image analysis and optical reflected light, Geological Society of America Abstracts with Programs, 35/6, 73, 2003

Nussbaum, S., Niemeyer, I. and Canty, M.J., Feature Recognition in the Context of automated Object-Oriented Analysis of Remote Sensing Data monitoring the Iranian Nuclear Sites, Proceedings of Optics/Photonics in Security \& Defence, SPIE , 2005

Turcotte, D.L.,Fractals and Chaos in Geology and Geophysics, 2nd Edition. Cambridge University Press, 1997. 